\title{
A Key Management Scheme for Mobile Ad Hoc Networks Based on Threshold Cryptography for Providing Fast Authentication and Low Signaling Load
}

\author{
Hoang Nam Nguyen ${ }^{1}$ and Hiroaki Morino ${ }^{2}$ \\ ${ }^{1}$ The National Institute of Information and Communication Technology, \\ 4-2-1 Nukui-kitamachi, Koganei-city, Tokyo 184-8795, Japan \\ nguyen@ict.go.jp \\ ${ }^{2}$ Shibaura Institute of Technology, \\ 3-9-14 Shibaura, Minato-ku, Tokyo 108-8548, Japan \\ morino@sic.shibaura-it.ac.jp
}

\begin{abstract}
Providing secure communications is a crucial task for the success of future ubiquitous mobile communication systems. Using public key infrastructure (PKI) is considered as a good solution to fulfill the task. However, as mobile ad hoc networks (MANET) inherit unique characteristics such as dynamic topology, non-infrastructure architecture, centralized PKI architectures are not suitable for dynamic MANET. The use of distributed PKI models is more appropriate but requires additional modifications to adapt with network changes. In this paper, we introduce a novel key management scheme for MANET, which exploits advantages of threshold cryptography. The major innovative aspect of this scheme is the use of temporal substitute certificate authorities (SCA), which form a PKI model of multi SCA groups. Performance results obtained by computer simulation show that the proposed key management scheme can reduce the latency of authentication, certificate update delay and the signaling load.
\end{abstract}

\section{Introduction}

In mobile ad hoc networks (MANET), an ad hoc node operates as not only an end terminal but also an intermediate router. Data packets sent by a source node can reach to a destination node via a number of hops i.e. more than one node might be involved in forwarding packets from sources to destinations. MANET inherits unique properties such as arbitrary and dynamic network topology, user mobility and less robust wireless links. These properties bring many significant technical challenges ranging from the physical layer to the application layer for radio resource allocation, QoS control, medium access control (MAC) protocol, routing and security. Various research efforts have been carried out aiming to provide QoS guaranteed and secure communications in MANET. To provide secure communication, each MANET has to achieve security requirements in terms of availability, confidentiality, integrity, authentication and non-reputation [1]. While designing security mechanisms for MANET, following features of MANET should be taken into account: weak-secure wireless link, user roaming, dynamic topology and huge number of nodes. Efficient security schemes should be distributed to achieve high survivability. In MANET, 
routing is one of the most important functions for delivering data between mobile nodes. Routing protocols in MANET are suffered various type of security threats and attacks which can come from external malicious nodes or internal compromised MANET nodes $[1,2]$. In order to protect routing information, routing packets have to be coded by using a suitable public-key mechanism $[3,4,5]$.

In the paper, we propose a novel key management scheme based on threshold cryptography theory for MANET. The scheme is differed to other existing schemes in terms of using temporal substitute certificate authorities (SCA). Nodes, which have more resources denoted as strong nodes, can be selected to operate as temporary CA forming multi-groups of trust servers in the network area. The use of multi SCA groups is aiming to provide short authentication delay and low signaling load. Performance evaluation obtained by computer simulation show that by using multi SCA-groups, the system is able to provide fast certificate update and low signaling load. In the next section, recent proposals for public key management schemes are presented and concepts of threshold cryptography are given. In section 3, our proposed key management model is presented where a new PKI model is introduced and authentication processes are described. Section 4 discusses the issues of a location-based SCA allocation scheme. Performance evaluation is presented in the next section. Finally, conclusion remarks are given in the last section.

\section{Related Works}

Two principles of existing key management in MANET are node participation and usage of trusted third parties [8] which are corresponding to certificate chain and virtual $C A$ approaches. The certificate chain approach [7] requires participated nodes to have strong processing capabilities. When many nodes participate to an authentication chain, the system is more vulnerable resulting in the trade-off between the number of participated nodes and security. The usage of trusted third parties can provide the guarantee of nodes trust i.e. the authentication provided by authority entities has higher level of confidence. In MANET, the central authority might be a target for DoS and compromising attacks. When mobile ad hoc nodes locate far from the central authority, their connections might be not available.

In a threshold cryptography scheme $(n, k)$, a public/private key pair $\left(\mathrm{P}_{\mathrm{K}}, \mathrm{S}_{\mathrm{K}}\right)$ of the network is generated where the $\mathrm{P}_{\mathrm{K}}$ is distributed to all nodes in the networks [9]. The private key $\mathrm{S}_{\mathrm{K}}$ is divided to $n$ parts so call secret shares so that if $k$ shares are combined, the private key is created. The nodes storing the secret shares are called as share servers or distributed certificate authorities. Public key management schemes proposed in [1] are purely based on threshold cryptography theory with enhanced properties. Authors have shown that by using distributed share servers, mobile ad hoc networks will have higher availability, higher fault tolerance and less vulnerability. A cluster-based security architecture for MANET exploiting threshold cryptography has been proposed in [6]. In this architecture, the network private key is distributed over cluster heads (CHs). When a new node enters the network, if it receives a beacon signal of a $\mathrm{CH}$, it will perform a log-on procedure to become a member of the cluster. Otherwise, the node can form a new cluster and become its own cluster head. When a $\mathrm{CH}$ leaves or joins the network, the secret shares have to be renewed. The paper showed good performance of this architecture under a small network size. 
A fully self-organized key management scheme has been proposed in [7] in which users issue certificates for each other based on their personal known information and relationship. Each user maintains a local certificate repository which stores certificates of its neighbors. When two users want to verify their public keys of each other, they try to find an appropriate chain of certificates. The scheme is suitable to open mobile ad hoc networks. However it requires particular computing power of nodes and consumes time and bandwidth. In [8] both threshold cryptography and trust chain are exploited in order to gain both high security requirements as well as the deployment for large scale MANET. The composite key management scheme introduces a security metric so called confidence value which defines the security level of certificate issuer. Certificates can be issued by CAs and nodes who can participate to authentication, denoted as participant nodes. CAs issue certificates to nodes those are connected directly where as participant nodes issue certificate to their neighbors. When a node tries to find a certificate chain to other node, the most appropriate chain is selected based on the concurrent confidence value of available certificate chains.

Existing key management schemes based on threshold cryptography do not consider issues of scalability, signaling load and authentication latency. In a large scale MANET, CAs can locate very far from nodes resulting in long authentication latency. When the number of nodes increases, high signaling traffic occurs. Our research objectives are to design a threshold cryptography based PKI scheme for MANET which can reduce authentication latency and signaling load.

\section{Public-Key Management Architecture}

The system model of managed open mobile ad hoc networks is shown in Fig. 1 where distributed authority entities are deployed. There are several types of ad hoc network nodes which have different node properties. Fixed and mobile stations have more capabilities of information processing and radio resource than other nodes. The stations can act as gateways connecting the ad hoc network to other external networks via wired or wireless (satellite) links. These stations can perform functionalities of routing and security. There are two classes of nodes for high power processing nodes denoted as "strong" nodes and low power processing nodes in the network.

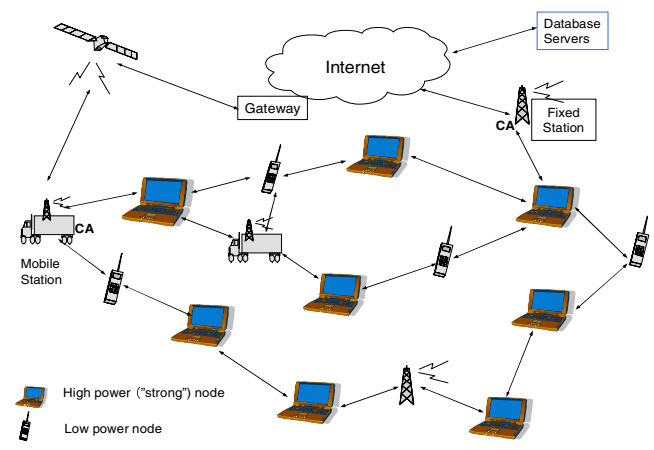

Fig. 1. System model 


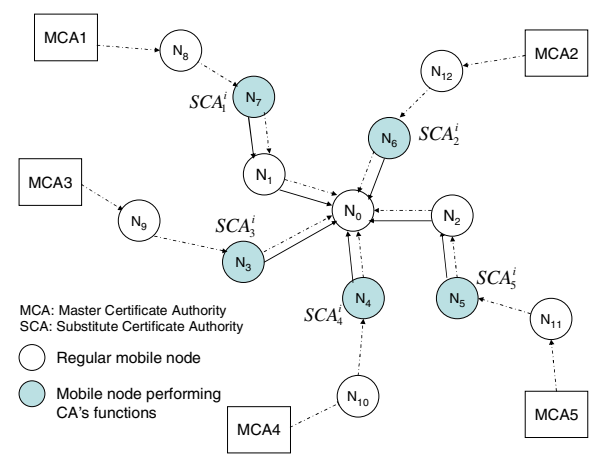

Fig. 2. Public-key management model

A novel public-key management model is shown in Fig. 2. At a given time, the network uses a pair of public and private keys $\left(\mathrm{P}_{\mathrm{K}}, \mathrm{S}_{\mathrm{K}}\right)$. Every node knows the public key $\mathrm{P}_{\mathrm{K}}$ of the network whereas the private key $\mathrm{S}_{\mathrm{K}}$ maintained by certificate authorities is used to sign certificates which prove the legal public keys of other nodes. In order to provide fast authentication and certificate update and reduce signaling load, the public key model exploits temporal substitute certificate authority (SCA) dynamically. End nodes, which have enough processing capability and resource to perform digital signature based functions, can act as SCA for a certain period. Each $\mathrm{MCA}_{\mathrm{j}}$ has a set of $(\mathbf{m}+1)$ secret shares $\left(\mathrm{S}_{\mathrm{K}}{ }^{0 \mathrm{j}}, \mathrm{S}_{\mathrm{K}}{ }^{1 \mathrm{j}} \ldots \mathrm{S}_{\mathrm{K}}{ }^{\mathrm{mj}}\right)$ where $\mathrm{S}_{\mathrm{K}}{ }^{0 \mathrm{j}}$ is used by the $\mathrm{MCA}_{\mathrm{j}}$. The other $\mathrm{S}_{\mathrm{K}}{ }^{\mathrm{ij}}$ can be distributed to corresponding $\mathrm{SCA}_{\mathrm{j}}{ }^{\mathrm{i}}$. That means there can be $\mathbf{m}$ different groups of SCA simultaneously where with the combination of $\mathbf{k}$ secret shares $S_{K}{ }_{\mathrm{K}}$ among a group, the private key $S_{K}$ is created.

Assume the nodes $\mathrm{N}_{3}$ to $\mathrm{N}_{7}$ are selected by MCAs to work temporarily as SCA for a certain period. When the node $\mathrm{N}_{0}$ enters the network, it can broadcast an authentication request and receive the first reply from node $\mathrm{N}_{3}$. and/or other nodes to inform about the list of available SCA. The $\mathrm{P}_{\mathrm{K}-\mathrm{N} 0}$ is sent to all $\mathrm{SCA}_{\mathrm{j}}^{\mathrm{i}}$ and waits for encrypted information signed by secret shares stored in the $\mathrm{SCA}_{\mathrm{j}}^{\mathrm{i}}$ denoted as $\left(\mathrm{N}_{0}, \mathrm{P}_{\mathrm{K}-\mathrm{N} 0}\right.$, $\mathrm{t}_{\mathrm{f}}, \mathrm{S}_{\mathrm{K}}{ }_{\mathrm{ij}}$ ). When the new node $\mathrm{N}_{0}$ has $\mathrm{k}=3$ encrypted information's components, it can create a certificate for the node signed by the private key $\left(\mathrm{N}_{0}, \mathrm{P}_{\mathrm{K}-\mathrm{N} 0}, \mathrm{t}_{\mathrm{f}}, \mathrm{S}_{\mathrm{K}}\right)$. The authentication delay is significantly reduced comparing with that of the conventional scheme, where nodes have to connect with only MCAs for authentication. MCAs have to compute secret key's fragments, update secret shares to all SCAi and cooperate each other to setup/release SCAs. MCAs/SCAs periodically broadcast their identity and certificates $\left(\mathrm{Cert}_{\mathrm{MCA}} / \mathrm{Cert}_{\mathrm{SCA}}\right)$ which prove that the nodes have legal rights to perform authentication process. Regular nodes store information of certificate authorities who can maintain node's certificate update. They have to perform the function for combining certificate share in order to create certificate. Because nodes have different roles, following types of certificates are needed: $\operatorname{Cert}_{\mathrm{MCA}}=\left\{\left(\mathrm{MCA}_{\mathrm{ID}}\right.\right.$, $\mathrm{P}_{\mathrm{MCA}}$, "MCA node" $\left.), \mathbf{S}_{\mathbf{K}}\right\}, \mathrm{Cert}_{\mathrm{SCA}}=\left\{\left(\mathrm{SCA}_{\mathrm{ID}}, \mathrm{P}_{\mathrm{SCA}}\right.\right.$, "SCA node", time $\left.\left.\mathrm{exp}\right), \mathbf{S}_{\mathbf{K}}\right\}$ and Cert $_{\text {node }}$ $=\left\{\left(\right.\right.$ node $_{\text {ID }}, \mathbf{P}_{\text {node }}$, "trust level", time $\left.\left.e_{\text {exp }}\right), \mathbf{S}_{\mathbf{K}}\right\}$ 


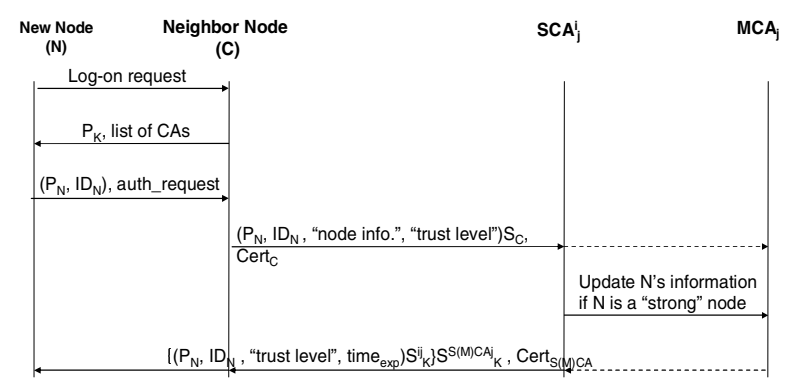

Fig. 3. Log-on authentication process

As shown in Fig. 3, when a new node $\mathrm{N}$ enters the mobile ad hoc network, it selects the neighbor who has the strongest signal strength and exchange information of network public key and the list of CAs. Then the node $\mathrm{N}_{0}$ sends an authentication request to this node. The neighbor node can evaluate the trust level of the new node according to the relationship between these nodes. The neighbor node adds the information of new node's trust level to this request. There are different scenarios where authentication is performed either by MCAs or by SCAs. When CAs receive this request, they will create certificate shares, sign by their private keys $\left(\mathrm{S}^{\mathrm{CA}_{-} \mathrm{j}}\right)$ and then send them to the new node. When the new node collects enough certificate shares, it can combine them and create its certificate. A certificate authority sends an appropriate certificate share together with the CA's certificate in order to avoid malicious nodes behaving as fake CAs. The new node can verify the CA's certificate and get the public key of the CA to decrypt the certificate share.

During its lifetime, the node $\mathrm{N}$ needs to update its certificate according to the validity time set by certificate authority. When a node moves within the network, its location might change frequently. The node needs to update the list of the nearest SCAs in order to obtain fast certificate update. The nodes exchange information of SCAs with each other in order to find the most appropriate SCAs for updating certificate. Each node can store the information of several SCA groups. When a node needs to update its certificate, the node can select a CAs group to ask for updating certificate. In order to reduce the signaling load, nodes can exchange information of SCA every long enough period.

\section{Location-Based SCA Allocation}

A possible and efficient SCA allocation approach is to allocate strong nodes as SCAs based on their location information. At the moment of time, mobile devices are easily equipped a GPS technology. Users can update their location information to gateway stations who also can act as MCAs. By periodically updating node's location information, MCAs can roughly draw a map of user distribution. Based on the database, MCAs can select strong nodes to work as SCAs efficiently. When a MANET is deployed to a remote area, the coverage area's geographical information of the MANET can be roughly identified and stored in MCAs. The MCAs can divide the coverage area into a number of cells for the purpose of key management. These 
cells can have different sizes and are assigned certain index. The geographical map and information of cells are stored in all MCAs and can be loaded into SCAs.

When a new node enters the network, the node performs authentication and update information of its location and node properties to MCAs. The node will receive geographical cell information from MCAs. Hence when nodes move within the network, they know which cell they locate. If MCAs consider this node as a candidate node for allocating as a SCA, this node's information (ID, location, resource) is stored in MCAS. Each MCA in $_{\mathrm{i}}$ selects a "strong" node in each cell. Strong nodes having more resources (bandwidth, CPU power) are given higher priority to become a SCA. In order to avoid the case that a strong node is selected by two MCAs for a cell, MCAs exchange each other the list of nodes who are acting as SCAs (node_ID, SCA_group_ID, SCA_ID).MCA sends SCA_allocation_request to a selected node and wait for the reply. If this node is available (not yet being acts as a SCA), SCA_allocation_reply is sent to the MCAi. Then Cert $_{\mathrm{SCA}}$ and a secret share are created and then sent to the node. When performing SCA's secret share update and maintenance, MCAs are responsible to cooperate with each other to update certificate of SCA $\left(\mathrm{Cert}_{\mathrm{SCAs}}\right)$. MCAs calculate new secret shares and deliver them to SCAs accordingly. The expiration time is the same for all SCAs belonging to a SCA_group. Certificates of the SCAs belonging to different SCA_group can have different expiration times.

SCA handover and release process is performed periodically i.e. after every certain period of time, the MCAs will update the secret shares for the SCAs in cell $\mathbf{i}$ and perform SCA handover at the same time. The strong node, who is acting as a SCA of a cell but locates in another cell, will be revoked the SCA rights. The corresponding MCA of the SCA will select another strong node located in the cell $\mathbf{i}$ to act as a new SCA for the cell. Secret share calculation is not the major aspect of this paper therefore the detailed issues of threshold cryptography are out of our scope. Mathematic issues of secret share calculation and construction without the need of trusted dealers have been well described in [9]. In our proposed scheme, MCAs are going to calculate and update secret shares for themselves and for SCAs periodically. The share refreshing process performed for a group $\mathbf{i}$ is described briefly as follows:

- A set of $\mathbf{k}$ MCAs will involve in the share refreshing process. Each MCA generate a set of key shares $\left(\mathrm{S}_{\mathrm{i}}^{\mathrm{x} 1}, \mathrm{~S}_{\mathrm{i}}^{\mathrm{x} 2} \ldots \mathrm{S}_{\mathrm{i}}^{\mathrm{xn}}\right)$ and delivers a $\mathrm{S}_{\mathrm{i}}^{\mathrm{xj}}$ to the corresponding $\mathrm{MCA}_{\mathrm{j}}$.

- Each $\mathrm{MCA}_{\mathrm{j}}$ calculate a share $\mathrm{S}_{\mathrm{K}}{ }_{\mathrm{ij}}$ by the following equation: $S_{K}^{i j}=\sum_{x=1}^{k} S_{i}^{x j}$

\section{Evaluate the Efficiency of This PKI Model}

Assume that the coverage of simulated ad hoc networks is a square of network size $D_{N}$ where users are uniformly generated. MCAs divide the network coverage to $\mathbf{m}$ homogeneous square cells which have the cell size of $D_{C}$. The MCAs will allocate a group of SCA for each cell i.e. the $\mathrm{MCA}_{\mathrm{j}}$ will allocate a $\mathrm{SCA}_{\mathrm{j}}^{\mathrm{i}}$ in the cell $\mathbf{i}$, as shown in Fig. 4. Assume that node $A$ wants to send packets to node $E$ which is out of the coverage of node A. Node A can connect directly with node B, C and D. We assume that signaling packets are delivered from $\mathrm{A}$ to $\mathrm{E}$ with a minimal number of hops i.e. A->D- $>$ E. With this assumption, the delay of signaling packets transmitting between MCA/SCA and nodes depends on the distance between them. If we assume that nodes 


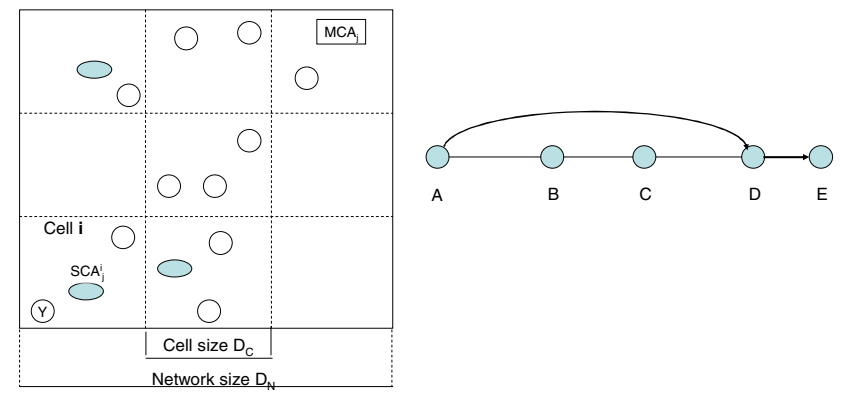

Fig. 4. Geographical map and packet routing

have the same maximum transmission distance $\left(\mathrm{d}_{\max }\right)$, a signaling packet delivered from a CA to nodes $(\mathrm{Y})$, those are far from each other with the distance of $\mathrm{d}_{\mathrm{CA}_{-} \mathrm{Y}}$, can be transmitted roughly via $\left(\mathrm{d}_{\mathrm{CA}_{-} \mathrm{Y}} / \mathrm{d}_{\max }+1\right)$ hops.

Fixed MCAs are generated and their positions are uniformly distributed. After that, new nodes are generated uniformly within the network coverage. In the proposed PKI model, there are $\mathrm{N}_{\text {S-node }}$ strong nodes among generated nodes. The MCAs will scan each cell to find if there are more than $\mathbf{n}$ strong nodes in the cell and then will select $\mathbf{n}$ of them as SCAs. Mobile nodes move within the network with a particular mobility model (e.g. random way point). When strong nodes move to a new cell, they will update their location information to all MCAs. In our proposed PKI model, when a SCA of cell $\mathbf{i}$ moves to another cell, it updates its new location information to all MCA. SCA handover is not performed immediately i.e. the strong node is still operating as a SCA for the previous cell for a given time.

A network area is a square of $3000 \mathrm{mx} 3000 \mathrm{~m}$ is simulated where the maximum transmission distance of a node is assumed $300 \mathrm{~m}$. In most simulation scenarios if not specifically mentioned, the proposed schemes (fixed or mobile SCA) divide the networks into 16 square cells and have 100 strong nodes and exploit threshold cryptography of $(5,3)$. Users mobility model is random waypoint model without

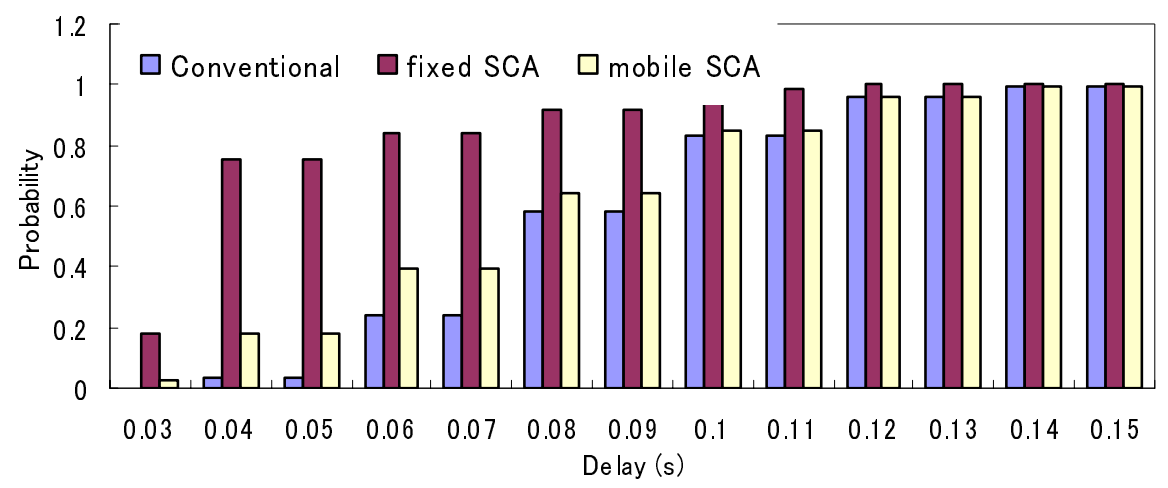

Fig. 5. Delay's pdf of certificate update of different schemes for 1000 nodes, $(5,3)$ threshold 


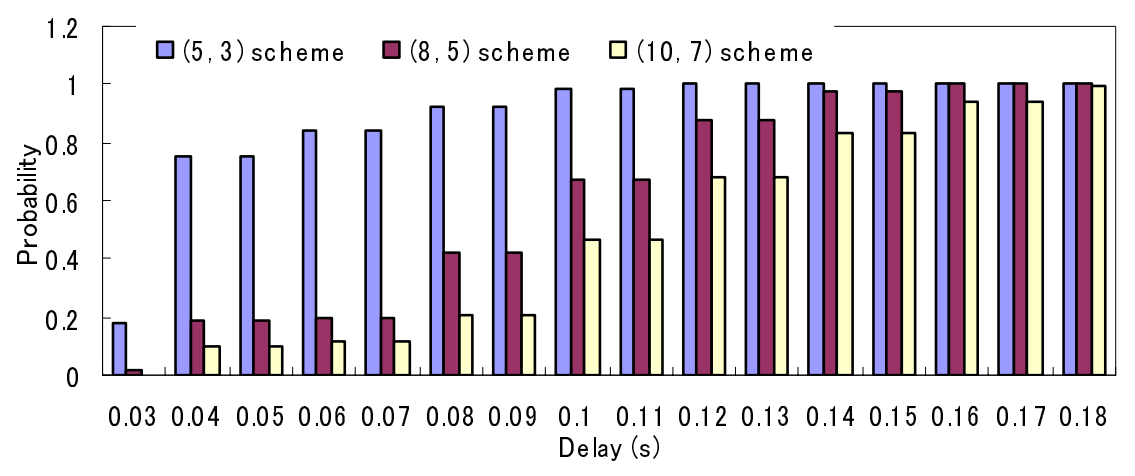

Fig. 6. Delay's pdf of certificate update: fixed-SCA scheme, 1000 nodes, different threshold

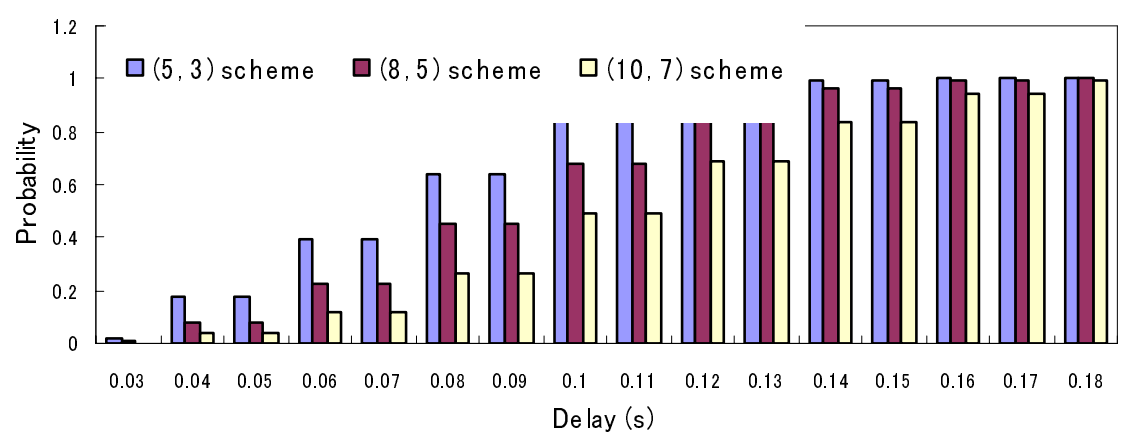

Fig. 7. Delay's pdf of certificate update of mobile-SCA scheme for 1000 nodes

pause time where user speed is varied between $1 \mathrm{~m} / \mathrm{s}$ to $10 \mathrm{~m} / \mathrm{s}$. Without loss of generality, we select the certificate update period is 5 minutes whereas share update is 15 minutes. Delay of a transmission hop is assumed 10ms. We compare the performance of three PKI schemes: conventional threshold cryptography, proposed scheme with fixed strong nodes (fixed SCA scheme) and the proposed scheme with mobile strong nodes (mobile SCA scheme).

Fig. 5 shows that the fixed SCA scheme is the best PKI scheme in terms of certificate update delay. It can provide nearly $20 \%$ of certificate update trials with delay less than $0.02 \mathrm{~s}$ (equivalent to 2 hops). It can provide more than $90 \%$ of updates with delay less than $0.09 \mathrm{~s}$. The mobile SCA scheme outperforms the conventional scheme when delay is lower than $0.09 \mathrm{~s}$. In the mobile SCA scheme, many trials get longer delay more than $0.09 \mathrm{~s}$. That is because when the strong nodes move, there are some time a cell has not enough SCAs. The nodes of this cell have to get certificate update from MCAs resulting in longer delay. Generally, by applying SCAs, the system will significantly reduce authentication latency. In Fig. 6 and 7, the certificate update delay for fixed SCA and mobile SCA schemes are presented, respectively for different threshold CA configuration. With more CA servers, system security is 


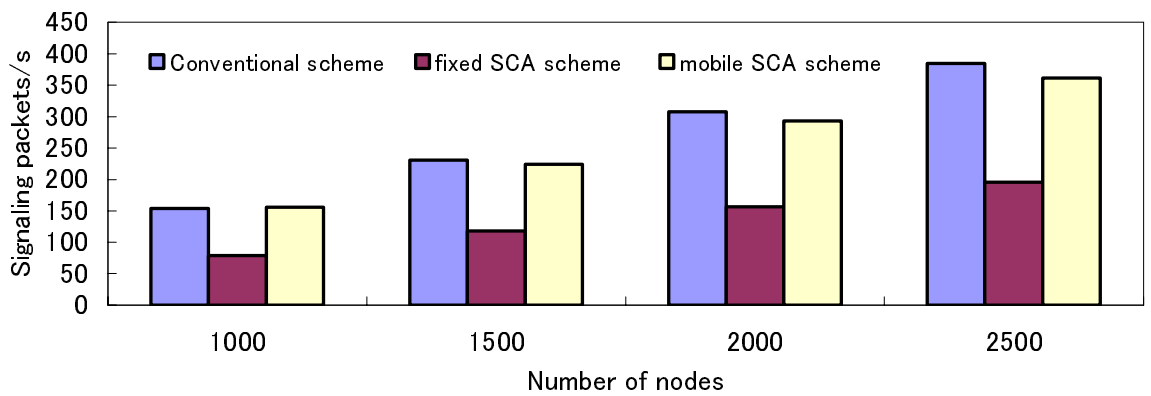

Fig. 8. Signaling load of different schemes with 16 cells, threshold $(5,3)$

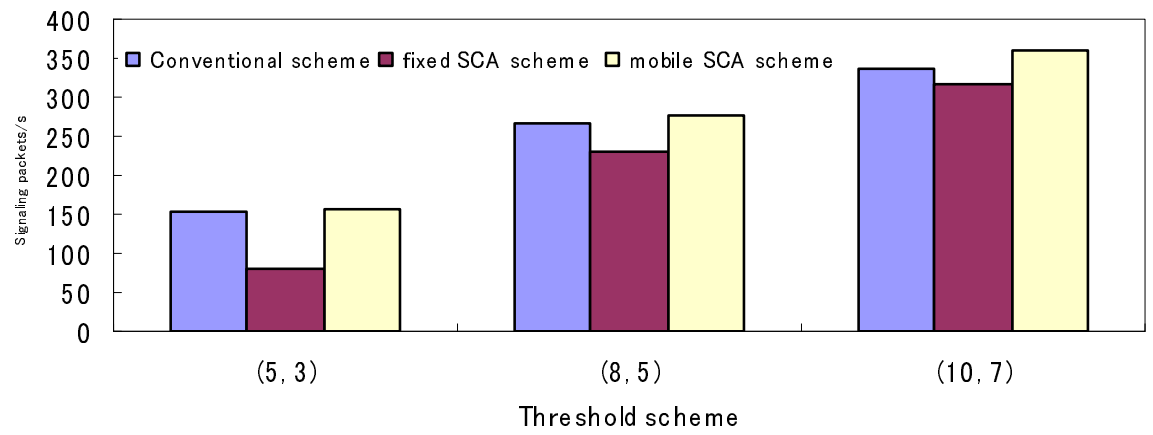

Fig. 9. Signaling load of different schemes: different threshold with 1000 nodes and 16cells

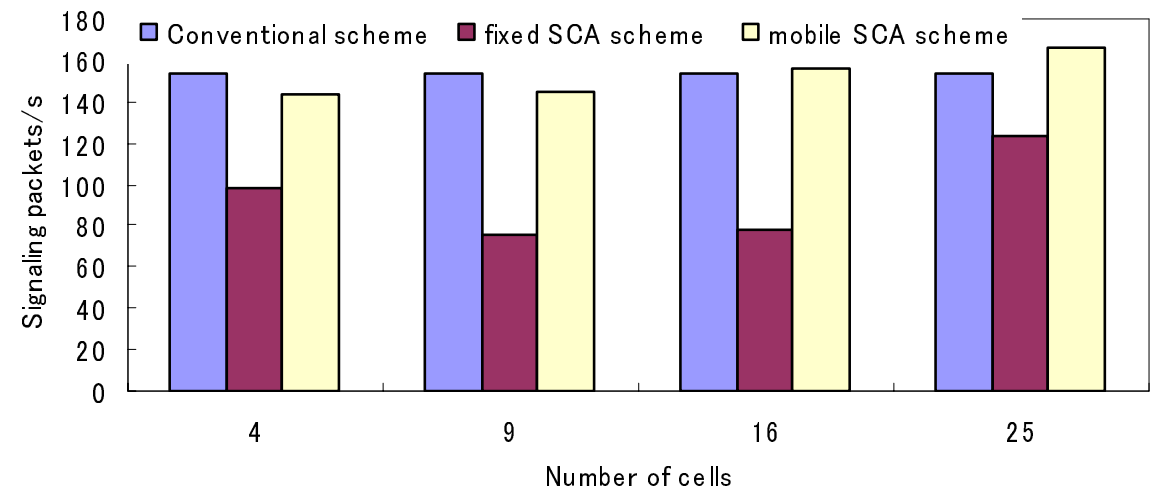

Fig. 10. Signaling load of different schemes for different cells: 1000 nodes, threshold $(5,3)$

increased. When the number of CA servers increases, the performance of proposed schemes will be decreased. That is because with a fixed number strong nodes and cells, when there are more CA servers, the scheme cannot provide SCAs to certain 
cells. That causes higher delay of certificate updates. Fig. 6 shows that the certificate update delay of the fixed SCA scheme decreases faster that that of the mobile SCA scheme. The reason is in the fixed SCA scheme, fixed strong nodes are allocated uniformly. With 100 strong nodes and 16 cells, there are average 6 strong nodes per cell. That means with higher threshold scheme of $(8,5)$ and $(10,7)$, many cells cannot get SCAs resulting in many long delay certificate updates. In mobile SCA scheme, when strong nodes move within the network coverage area, more strong nodes can locate in a cell. Therefore it causes higher probability that SCAs can be provided in the cell resulting in slow degradation of delay performance.

Fig. 8 to Fig. 10 show the signaling load of key management schemes for different simulation scenarios. In Fig. 8, threshold $(5,3)$ and 16 cells are simulated with different number of nodes. The fixed SCA schemes always provide low signaling load because fixed strong nodes do not need to update their locations and SCA handover is not necessary. When there are about 1000 nodes, the mobile SCA scheme needs signaling load similarly as that of conventional scheme. The reason is that the mobile SCA scheme has to update location of strong nodes and SCA handovers. Although it can provide low signaling load for certificate update, total signaling load of mobile SCA scheme is nearly equal to that of the conventional scheme. In Fig. 9, when different threshold configuration is applied, the fixed SCA scheme still will provide less signaling load than other schemes. However, as described above, more CA servers reduce the probability of successful SCA allocation in cells. Thus the signaling load of the fixed SCA scheme will increase fast. The mobile SCA scheme even provides more signaling load than the conventional scheme because it needs more signaling to update location of strong nodes when they move from a cell to another and more signaling load for share updates and SCA handover. Fig. 10 shows the signaling load of simulation scenarios with different cells. With the threshold (5, 3) and 1000 nodes, when the SCA-based schemes divide the network area to more cells, the signaling load of the mobile SCA scheme increases. When there are so many cells, its signaling load is even higher than that of the conventional scheme because it needs to update strong node location more frequently. The fixed SCA scheme provides higher signaling load in 4-cell scenario because, the distance between SCAs and nodes is longer resulting in more signaling for certificate updates. In the 25-cell scenario, not all cells can be provided SCAs thus resulting in more signaling load for certificate updates.

\section{Conclusions}

Using public key is an efficient solution to provide secure communication in mobile ad hoc networks. Due to the dynamic and large scale properties of MANET, providing efficient key management is a crucial task. In this paper, we have presented our public key management schemes based on threshold cryptography. Comparing with other variants of threshold cryptography, the novel proposed key management scheme exploits strong nodes as temporary substitute certificate authorities (SCA) in order to provide fast certificate update and low signaling load. Performance results show that generally the SCA-base key management schemes can gain more benefits than the conventional scheme in terms of delay and signaling load. However, there are still open research issues in terms of optimizing the SCA allocation and reducing the signaling load as well as the certificate update delay. Our future works are to optimize 
the performance of the proposed schemes and evaluate their performance under different application scenarios.

\section{References}

[1] L. Zhou, Z. J. Haas, "Securing ad hoc networks", IEEE Network, Vol. 13, No. 6, Nov. 1999, pp: $24-30$

[2] Y. C. Hu, A. Perrig, "A survey of secure wireless ad hoc routing”, IEEE Security \& Privacy Magazine, Vol. 2, No. 3, May-June 2004, pp: 28 - 39

[3] Y. C. Hu, A. Perrig, D. B. Johnson, "Ariadne: A Secure On-demand Routing Protocol for Ad Hoc Networks", in the Proc. Of $8^{\text {th }}$ Annual International Conference Mobile Computing and Networking (Mobicom 2002), ACM Press, 20002, pp. 12-23

[4] M. G. Zapata, N. Asokan, "Securing ad hoc routing protocols", in the Proc. Of ACM Workshop on wireless security (WiSe), ACM Press, 2002, pp: 1-10

[5] K. Sanzgiri et al., "A secure routing protocol for ad hoc networks", 10th IEEE International Conference on Network Protocols, 2002, 12-15 Nov. 2002, pp: 78 - 87

[6] M. Bechler, H-J. Hof, D. Kraft, F. Pahlke, L. Wolf, "A cluster-based security architecture for ad hoc networks", Twenty-third Annual Joint Conference of the IEEE Computer and Communications Societies, INFOCOM 2004, Vol. 4, 7-11 March 2004, pp: 2393 - 2403.

[7] S. Capkun, L. Buttyan, J. P. Hubaux, "Self-organized public-key management for mobile ad hoc networks", IEEE Transactions on Mobile Computing, Vol. 2, No. 1, Jan. 2003, pp: $52-64$

[8] S. Yi, R. Kravets, "Composite Key Management for Ad hoc networks", The First International Conference on Mobile and Ubiquitous Systems: Networking and Services, MOBIQUITOUS 2004, 22-26 Aug. 2004, pp: 52 - 61.

[9] Y. Desmedt, "Some recent research aspects of threshold cryptography", In: R. Okamoto eds. Information Security, LNCS 1396, Springer-Verlag, 1997, pp: 158-173. 